\title{
Quenching of $\mathrm{H} 2$ Vibrations in Ultracold $3 \mathrm{He}$ and 4He Collisions
}

\section{Citation}

Balakrishnan, N., R. C. Forrey, and A. Dalgarno. 1998. "Quenching ofH2Vibrations in Ultracold3Heand4HeCollisions." Physical Review Letters 80 (15) (April 13): 3224-3227. doi:10.1103/physrevlett.80.3224.

\section{Published Version}

doi:doi.org/10.1103/PhysRevLett.80.3224

\section{Permanent link}

http://nrs.harvard.edu/urn-3:HUL.InstRepos:30410805

\section{Terms of Use}

This article was downloaded from Harvard University's DASH repository, and is made available under the terms and conditions applicable to Other Posted Material, as set forth at http:// nrs.harvard.edu/urn-3:HUL.InstRepos:dash.current.terms-of-use\#LAA

\section{Share Your Story}

The Harvard community has made this article openly available.

Please share how this access benefits you. Submit a story.

\section{Accessibility}




\title{
Quenching of $\mathrm{H}_{2}$ Vibrations in Ultracold ${ }^{3} \mathrm{He}$ and ${ }^{4} \mathrm{He}$ Collisions
}

\author{
N. Balakrishnan, R. C. Forrey, and A. Dalgarno \\ Institute for Theoretical Atomic and Molecular Physics, Harvard-Smithsonian Center for Astrophysics, 60 Garden Street, \\ Cambridge, Massachusetts 02138
}

(Received 2 December 1997)

\begin{abstract}
We present results of quantum mechanical scattering calculations including full rovibrational coupling on the relaxation of vibrationally excited $\mathrm{H}_{2}$ molecules in collision with ultracold ${ }^{3} \mathrm{He}$ and ${ }^{4} \mathrm{He}$ atoms. The exothermic vibrational deactivation cross sections exhibit minima at translational temperatures near $10 \mathrm{~K}$ and show an inverse velocity dependence in accordance with Wigner's threshold law in the limit of zero kinetic energy. The corresponding rate coefficients exhibit minima before attaining constant values below $10^{-3} \mathrm{~K}$. The rate coefficients increase by 3 orders of magnitude when the initial vibrational level of the molecule is raised from $v=1$ to $v=10$. The results suggest that quasibound resonance states of ${ }^{3} \mathrm{HeH}_{2}$ and ${ }^{4} \mathrm{HeH}_{2}$ exist, associated with each excited vibrational state of $\mathrm{H}_{2}$, and that both the triatomic species ${ }^{3} \mathrm{HeH}_{2}$ and ${ }^{4} \mathrm{HeH}_{2}$ have one bound vibrational state. [S0031-9007(98)05812-8]
\end{abstract}

PACS numbers: 34.50.Ez, 33.70.-w

In several of the schemes that have been suggested for the creation of ultracold molecules [1-5], translationally cold molecules in high lying vibrational levels are produced as intermediaries. These highly excited molecules are themselves of interest because they open the possibility of measurements of exothermic reactions at ultracold translational temperatures and the experimental testing of threshold laws. In the conventional view of vibrational quenching [6] the process is driven by the derivative of the interaction potential with respect to intermolecular distance, and the rate coefficient is expected to decrease rapidly as the temperature decreases to zero. However, at some point the behavior of the rate coefficient must be modified by the influence of the long-range part of the interaction potential [7] and further modified by the requirement that the cross section satisfies the threshold law that it increases as the inverse of the velocity [8-12]. The zero temperature rate coefficient must be finite, though it may be very small.

We have set up and solved the close-coupling equations describing the scattering of $\mathrm{He}$ and $\mathrm{H}_{2}$ on a reliable potential energy surface in which a sufficient number of rotational and vibrational states of $\mathrm{H}_{2}$ has been included to secure convergence. We find quenching cross sections which pass through minimum values at collision energies between $10^{-2}$ and $10^{-3} \mathrm{eV}$ and tend to increase inversely as the velocity below energies of about $10^{-3} \mathrm{eV}$. The rate coefficients at ultralow temperatures increase by 3 orders of magnitude as the vibrational level is increased from $v=1$ to $v=10$.

In the total angular momentum representation of Arthurs and Dalgarno [13], the cross sections for transitions from an initial vibrational-rotational level labeled by quantum numbers $v j$ to a final level labeled by quantum numbers $v^{\prime} j^{\prime}$ can be expressed in terms of the corresponding $S$-matrix elements $[13,14]$

$$
\begin{aligned}
\sigma_{v, j \rightarrow v^{\prime}, j^{\prime}}= & \frac{\pi}{(2 j+1) k_{v j}^{2}} \sum_{J=0}^{\infty}(2 J+1) \\
& \times \sum_{l=|J-j|}^{|J+j|} \sum_{l^{\prime}=\left|J-j^{\prime}\right|}^{\left|J+j^{\prime}\right|}\left|\delta_{j j^{\prime}} \delta_{l l^{\prime}} \delta_{v v^{\prime}}-S_{j j^{\prime} l l^{\prime} v v^{\prime}}^{J}\right|^{2},
\end{aligned}
$$

where $J$ is the total angular momentum quantum number and $l$ and $l^{\prime}$ are, respectively, the initial and final orbital angular momentum quantum numbers. The wave vector for the incoming channel is defined as $k_{v j}=$ $\sqrt{2 \mu\left(E-\epsilon_{v j}\right)} / \hbar$ where $E$ is the total energy, $\epsilon_{v j}$ is the eigenenergy of the initial rovibrational state, and $\mu$ is the three-body reduced mass. The total exoergic de-excitation cross section to all open rovibrational channels is the sum

$$
\sigma_{v j}^{\mathrm{exo}}=\sum_{j^{\prime}, v^{\prime}<v} \sigma_{v, j \rightarrow v^{\prime}, j^{\prime}}
$$

The rate coefficient for a given transition is obtained by averaging the appropriate cross section over a Boltzmann distribution of velocities of the incoming atom at a specified temperature $T$ :

$$
\begin{aligned}
r_{v, j \rightarrow v^{\prime}, j^{\prime}}(T)= & \left(8 k_{B} T / \pi \mu\right)^{1 / 2} \frac{1}{\left(k_{B} T\right)^{2}} \\
& \times \int_{0}^{\infty} \sigma_{v, j \rightarrow v^{\prime}, j^{\prime}}\left(E_{k}\right) \exp \left(-E_{k} / k_{B} T\right) E_{k} d E_{k},
\end{aligned}
$$

where $E_{k}=\hbar^{2} k_{v j}^{2} /(2 \mu)$ is the kinetic energy of the incoming atom and $k_{B}$ is the Boltzmann constant.

The cross sections at zero energy may be characterized by scattering lengths. If more than one channel is open, the scattering lengths are complex quantities $a_{v j}=$ $\alpha_{v j}-i \beta_{v j}$ [15]. The elastic cross section for level $v j$ is given at zero energy by

$$
\sigma_{v j}^{e l}=4 \pi\left(\alpha_{v j}^{2}+\beta_{v j}^{2}\right)
$$




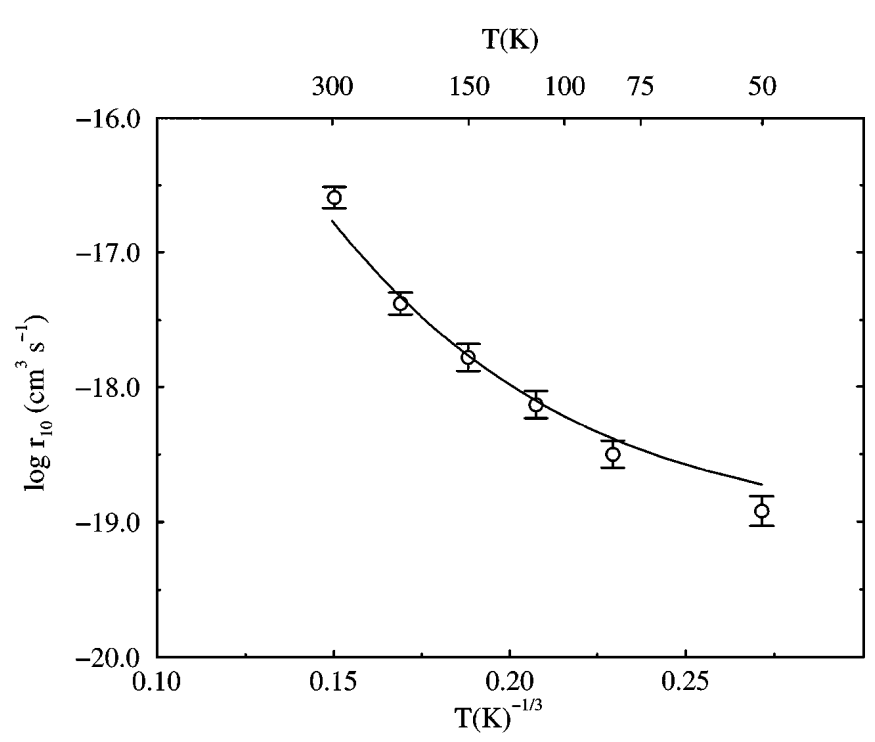

FIG. 1. Rate coefficient for the relaxation of the $v=1$ state of para- $\mathrm{H}_{2}$ in collision with ${ }^{4} \mathrm{He}$. The solid line is the present result obtained from $\sigma_{10}^{\text {exo }}$ and the symbols with error bars are the experimental results of Audibert et al. [24].

and the total inelastic cross section by

$$
\sigma_{v j}^{i n}=\sum_{v^{\prime} j^{\prime}} \sigma_{v j \rightarrow v^{\prime} j^{\prime}}=4 \pi \beta_{v j} / k_{v j} .
$$

The corresponding zero temperature rate coefficient is

$$
r_{v j}=4 \pi \hbar \beta_{v j} / \mu \text {. }
$$

The coupled equations for the radial motion obtained by using the expansion for the wave function in the time-independent Schrödinger equation were solved using the nonreactive scattering program MOLSCAT [16]. We

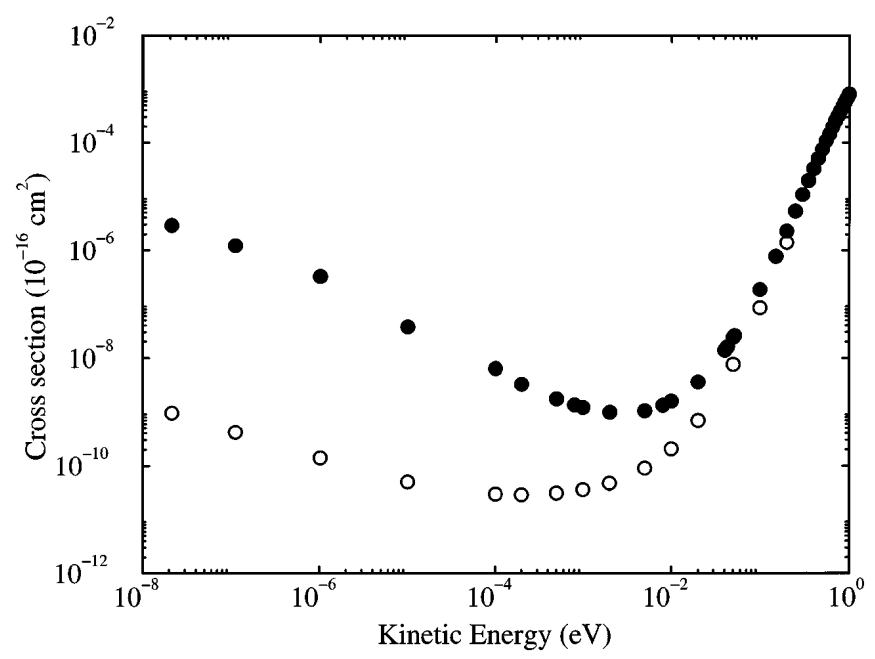

FIG. 2. Exothermic vibrational relaxation cross section $\sigma_{1,0 \rightarrow 0,0}$ for ${ }^{4} \mathrm{He}-\mathrm{H}_{2}$ collisions as a function of kinetic energy. Filled circles are the results obtained using the full interaction potential and the open circles are the results obtained from a calculation in which the long-range part of the potential is omitted.

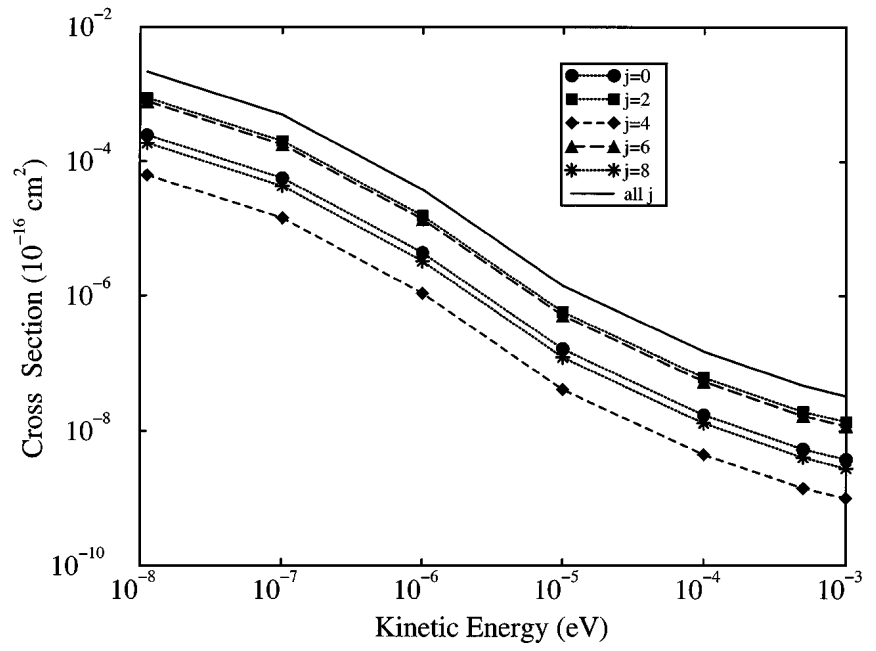

FIG. 3. Rovibrational transition cross sections $\sigma_{1,0 \rightarrow 0, j}$ in $\mathrm{H}_{2}$ in ${ }^{3} \mathrm{He}$ collisions as a function of kinetic energy.

obtained the diatomic vibrational wave functions used in generating the coupled equations as expansions in Hermite polynomials with the diatomic $\mathrm{H}_{2}$ potential of [17].

Close-coupling calculations for collisions of ${ }^{4} \mathrm{He}$ with $\mathrm{H}_{2}$ have been carried out using various potential energy surfaces and scattering formulations [18-22], and rate coefficients have been reported for temperatures above $50 \mathrm{~K}$. We have adopted the more recent potential energy surface (PES) of Muchnick and Russek [23]. Figure 1 compares our calculations with the experimental data of Audibert et al. [24] on the transition from the $v=1$ vibrational level of $\mathrm{H}_{2}$ to the $v=0$ level caused by collisions with ${ }^{4} \mathrm{He}$ for temperatures between 300 and $50 \mathrm{~K}$. The close agreement suggests that the PES [23] is reliable. The measured rate coefficients fall very quickly with decreasing temperature.

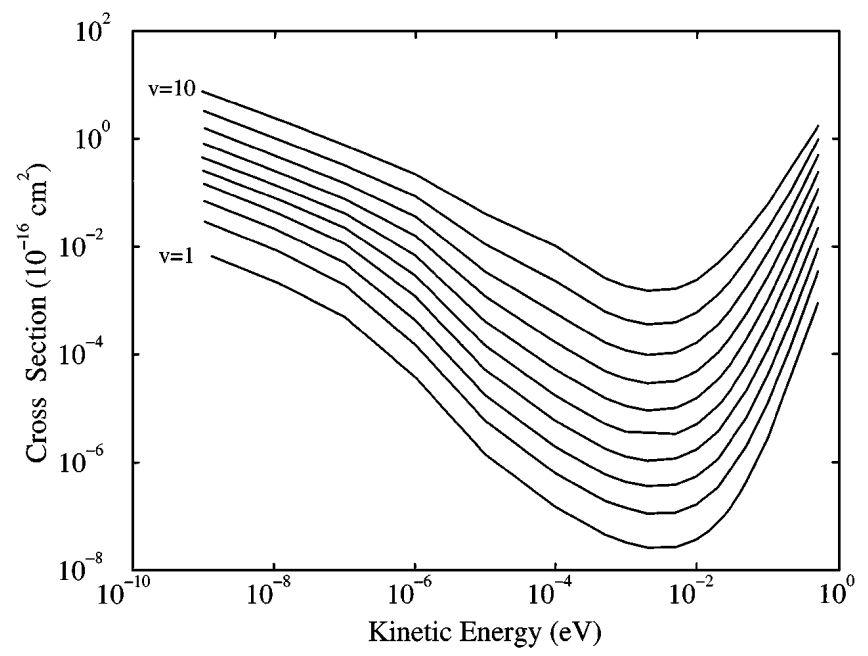

FIG. 4. Total quenching cross sections $\sigma_{v 0}^{\text {exo }}$ as a function of kinetic energy of incoming ${ }^{3} \mathrm{He}$ atoms for different vibrational levels of $\mathrm{H}_{2}$. The curves correspond to cross sections for $v=1$ to $v=10$ in ascending order. 


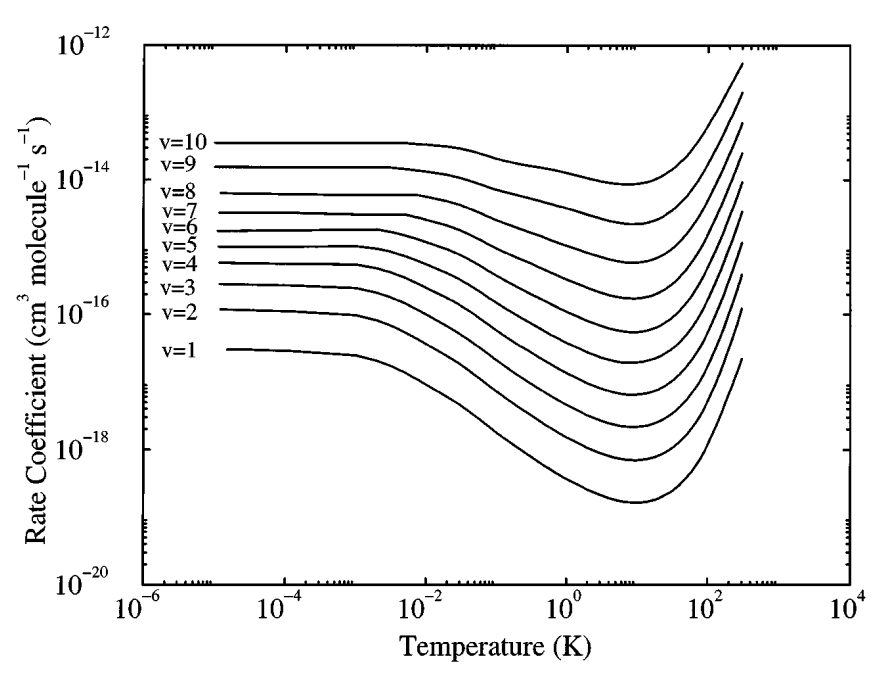

FIG. 5. Total vibrational relaxation rate coefficients for the deactivation of $\mathrm{H}_{2}(v, 0)$ in collisions with ${ }^{3} \mathrm{He}$ as a function of temperature for different vibrational levels of the molecule.

In Fig. 2 we show the 1-0 cross section extended to very low incident energies. The cross section decreases to a low minimum value at about $2 \times 10^{-3} \mathrm{eV}$ and then increases as the energy decreases further, and eventually conforms to an inverse velocity dependence in accordance with Wigner's law. The cross sections obtained when we suppress the long-range attractive forces are also shown in Fig. 2. It is clear that the values of the low energy cross sections are determined by the long-range van der Waals interaction, but even in the absence of the van der Waals interaction the cross section passes through a minimum and increases inversely as the velocity, though the values are reduced by several orders of magnitude.

The cross sections for quenching of the $v=1, j=0$ rotational level into the individual rotational levels of the $v=0$ vibrational state are shown in Fig. 3 at energies in the threshold region. Rotational levels beyond $j=8$ are not accessible at low energies. The weakness of the anisotropy of the interaction potential causes small changes in $j$ to be favored, but the final distribution is modified by the enhancement in scattering into high $j$ levels caused by the matching of the momenta of the incoming and outgoing waves which is closest for the least exothermic channel $j=8$.

In Fig. 4, we show the total quenching cross sections as functions of the kinetic energy of incoming ${ }^{3} \mathrm{He}$ atoms for the initial $j=0$ rotational state of $\mathrm{H}_{2}$ and vibrational states $v=1$ to $v=10$. The cross sections all pass through minima in the region $10^{-2} \mathrm{eV}$ and increase inversely with velocity as the energy tends to zero. At any given energy, the cross sections increase with increasing vibrational quantum number. The vibrational relaxation takes place predominantly by single quantum transitions $\Delta v=1$, in contrast to the case of $\mathrm{H}-\mathrm{H}_{2}$ for which multiquantum transitions are probable [25].

The rate coefficients for ${ }^{3} \mathrm{He}-\mathrm{H}_{2}$ are presented in Fig. 5. They tend to constant values at zero temperature. The zero temperature values increase from $3 \times 10^{-17} \mathrm{~cm}^{3} \mathrm{~s}^{-1}$ for $v=1$ to $3.6 \times 10^{-14} \mathrm{~cm}^{3} \mathrm{~s}^{-1}$ for $v=10$.

The real and imaginary parts of the scattering lengths for ${ }^{3} \mathrm{He}$ and ${ }^{4} \mathrm{He}$ colliding with $\mathrm{H}_{2}(v)$ are listed in Table I. Equations (1)-(6) may be used to calculate the zeroenergy elastic and total inelastic cross sections and rate coefficients. The real parts of the scattering lengths are large, suggesting that associated with each vibrational level of $\mathrm{H}_{2}$ is a bound state of $\mathrm{He}-\mathrm{H}_{2}$ lying energetically below the dissociation limit of the $\mathrm{He}-\mathrm{H}_{2}(v)$ complex. The state associated with $v=0$ is a true bound state. The states associated with $v>0$ are quasibound resonance states of $\mathrm{He}-\mathrm{H}_{2}$ and they may undergo vibrational predissociation into $\mathrm{He}+\mathrm{H}_{2}(v)$. Their predissociation lifetimes can be obtained from the imaginary parts of the scattering lengths using the expression [15]

$$
\tau_{v j}=\mu\left|a_{v j}\right|^{4} / 2 \hbar \alpha_{v j} \beta_{v j}
$$

The values of $\tau_{v 0}$ are listed in Table I. The lifetimes are much longer than the vibrational periods, indicating that the quasibound states are well defined. For the lowest bound states, we estimate binding energies of $0.0298 \mathrm{~cm}^{-1}$ for ${ }^{4} \mathrm{HeH}_{2}$ and $0.0016 \mathrm{~cm}^{-1}$ for ${ }^{3} \mathrm{HeH}_{2}$ but

TABLE I. Complex scattering lengths and lifetimes of the bound or quasibound $\mathrm{He} \cdots \mathrm{H}_{2}(v)$.

\begin{tabular}{crccccc}
\hline \hline & & & & & \\
& ${ }^{3} \mathrm{He} \cdots \mathrm{He}_{2} \cdots \mathrm{H}_{2}$ & \\
$v$ & $\alpha_{v 0}(\AA)$ & $\beta_{v 0}(\AA)$ & $\tau_{v 0}(s)$ & $\alpha_{v 0}(\AA)$ & $\beta_{v 0}(\AA)$ & $\tau_{v 0}(s)$ \\
\hline 0 & 95.6 & 0.0 & $\infty$ & 24.7 & 0.0 & $\infty$ \\
1 & 101.0 & $4.5 \times 10^{-7}$ & $2.2 \times 10^{-1}$ & 25.3 & $1.2 \times 10^{-8}$ & $1.4 \times 10^{-1}$ \\
2 & 98.8 & $1.8 \times 10^{-6}$ & $5.2 \times 10^{-2}$ & 25.5 & $5.2 \times 10^{-8}$ & $3.4 \times 10^{-2}$ \\
3 & 88.9 & $4.2 \times 10^{-6}$ & $1.6 \times 10^{-2}$ & 25.1 & $1.4 \times 10^{-7}$ & $1.2 \times 10^{-2}$ \\
4 & 74.7 & $8.7 \times 10^{-6}$ & $4.5 \times 10^{-3}$ & 24.3 & $3.8 \times 10^{-7}$ & $4.0 \times 10^{-3}$ \\
5 & 60.1 & $1.6 \times 10^{-5}$ & $1.3 \times 10^{-3}$ & 23.0 & $9.4 \times 10^{-7}$ & $1.4 \times 10^{-3}$ \\
6 & 47.5 & $2.7 \times 10^{-5}$ & $3.8 \times 10^{-4}$ & 21.4 & $2.3 \times 10^{-6}$ & $4.5 \times 10^{-4}$ \\
7 & 37.4 & $4.9 \times 10^{-5}$ & $1.0 \times 10^{-4}$ & 19.5 & $5.5 \times 10^{-6}$ & $1.4 \times 10^{-4}$ \\
8 & 29.5 & $9.3 \times 10^{-5}$ & $2.6 \times 10^{-5}$ & 17.5 & $1.4 \times 10^{-5}$ & $4.0 \times 10^{-5}$ \\
9 & 23.5 & $2.0 \times 10^{-4}$ & $6.2 \times 10^{-6}$ & 15.6 & $4.0 \times 10^{-5}$ & $1.0 \times 10^{-5}$ \\
10 & 18.8 & $4.6 \times 10^{-4}$ & $1.4 \times 10^{-6}$ & 13.7 & $1.2 \times 10^{-4}$ & $2.2 \times 10^{-6}$ \\
\hline \hline
\end{tabular}


the values are sensitive to the details of the potential energy surface.

This work was supported by the U.S. Department of Energy, Office of Basic Energy Sciences, Office of Energy Research (R.C.F.), and by the National Science Foundation, Division of Atmospheric Science (N. B.).

[1] J. M. Doyle, B. Friedrich, J. Kim, and D. Patterson, Phys. Rev. A 52, R2515 (1995).

[2] Y.B. Band and P.S. Julienne, Phys. Rev. A 51, R4317 (1995).

[3] B. Friedrich and D. R. Herschbach, Phys. Rev. Lett. 74, 4623 (1995).

[4] J. T. Bahns, W.C. Stwalley, and P.L. Gould, J. Chem. Phys. 104, 9689 (1996).

[5] R. Côté and A. Dalgarno, Chem. Phys. Lett. 279, 50 (1997).

[6] E. Wertz and G. Flynn, Annu. Rev. Phys. Chem. 25, 275 (1974).

[7] G. Ewing, Chem. Phys. Lett. 29, 253 (1978).

[8] W. R. Gentry, J. Chem. Phys. 81, 5737 (1984).

[9] T. Takayanagi, N. Masaki, K. Nakamura, M. Okamoto, and G. C. Schatz, J. Chem. Phys. 86, 6133 (1987).

[10] G. C. Hancock, C. A. Mead, D. G. Truhlar, and A. J. C. Varandas, J. Chem. Phys. 91, 3492 (1989).

[11] S. K. Gray and S. A. Rice, J. Chem. Phys. 83, 2818 (1985).

[12] D. W. Schwenke and D. G. Truhlar, J. Chem. Phys. 83, 3454 (1985).
[13] A. M. Arthurs and A. Dalgarno, Proc. R. Soc. London A 256, 540 (1963).

[14] W. A. Lester, Jr., in Dynamics of Molecular Collisions, edited by W.H. Miller (Plenum, New York, 1976), Part A, pp. 1-32.

[15] N. Balakrishnan, V. Kharchenko, R.C. Forrey, and A. Dalgarno, Chem. Phys. Lett. 280, 5 (1997).

[16] J.M. Hutson and S. Green, MOLSCAT computer code, version 14 (1994), distributed by Collaborative Computational Project No. 6 of the Engineering and Physical Sciences Research Council (UK).

[17] A. I. Boothroyd, W. J. Keogh, P. G. Martin, and M. R. Peterson, J. Chem. Phys. 104, 7139 (1996).

[18] M. H. Alexander, Chem. Phys. Lett. 38, 417 (1976).

[19] A. P. Clark, J. Phys. B 10, L389 (1977).

[20] A. W. Raczkowski, W. A. Lester, Jr., and W.H. Miller, J. Chem. Phys. 69, 2692 (1978).

[21] J. Schaefer and W.E. Köhler, Physica (Amsterdam) 129A, 469 (1985).

[22] M. J. Smith, S. Shi, and H. Rabitz, J. Chem. Phys. 91, 1051 (1989).

[23] P. Muchnick and A. Russek, J. Chem. Phys. 100, 4336 (1994).

[24] M. M. Audibert, C. Joffrin, and J. Ducuing, J. Chem. Phys. 61, 4357 (1974); M. M. Audibert, R. Vilaseca, J. Lukasik, and J. Ducuing, Chem. Phys. Lett. 31, 232 (1975); M. M. Audibert, R. Vilaseca, J. Lukasik, and J. Ducuing, Chem. Phys. Lett. 37, 408 (1976).

[25] N. Balakrishnan, R.C. Forrey, and A. Dalgarno, Chem. Phys. Lett. 280, 1 (1997). 